\title{
Bridging the gap to mesoscale radiation materials science with transient grating spectroscopy
}

\author{
Cody A. Dennett, ${ }^{1}$ Penghui Cao, ${ }^{1}$ Sara E. Ferry, ${ }^{1}$ Alejandro Vega-Flick,,${ }^{2,3}$ Alexei A. Maznev, ${ }^{2}$ Keith A. Nelson, ${ }^{2}$ \\ Arthur G. Every, ${ }^{4}$ and Michael P. Short ${ }^{1, *}$ \\ ${ }^{1}$ Department of Nuclear Science and Engineering, Massachusetts Institute of Technology, Cambridge, Massachusetts 02139, USA \\ ${ }^{2}$ Department of Chemistry, Massachusetts Institute of Technology, Cambridge, Massachusetts 02139, USA \\ ${ }^{3}$ Applied Physics Department, CINVESTAV-Unidad Mérida, Carretera Antigua a Progreso Km 6, \\ Cordemex, Mérida, Yucatán 97310, Mexico \\ ${ }^{4}$ School of Physics, University of the Witwatersrand, PO Wits 2050, South Africa
}

(Received 3 September 2016; revised manuscript received 25 October 2016; published 13 December 2016)

\begin{abstract}
Direct mesoscale measurements of radiation-induced changes in the mechanical properties of bulk materials remain difficult to perform. Most widely used characterization techniques are either macro- or microscale in nature, focusing on overall properties or overly small areas for analysis. Linking the atomic structure of irradiated materials directly with their radiation-affected properties remains one of the largest unmet challenges in radiation materials science. By measuring the change in surface acoustic wave speed as a function of relative orientation on metallic single crystals, we demonstrate that transient grating (TG) spectroscopy experiments have the sensitivity necessary to detect radiation-induced material property changes. We also show that classical molecular dynamics (MD) simulations can be used to accurately simulate orientation-based changes in surface acoustic wave speed in TG experiments, by comparing with experimental measurements and theoretical predictions. The agreement between theory, simulation, and experiment gives confidence in classical MD as a predictive tool to simulate defect-based changes in elastic properties, which cannot yet be fully treated by theory. This ability is of critical importance for the informed use of TG spectroscopy to measure material property changes induced by radiation damage, which may vary by amounts formerly too small for reliable in situ detection. Finally, our MD simulation framework is used to study the effect of an imposed vacancy population on the acoustic response of several materials. The results of these studies indicate that TG experiments are well suited to the ex situ and in situ study of radiation-induced material property changes.
\end{abstract}

DOI: 10.1103/PhysRevB.94.214106

\section{INTRODUCTION AND BACKGROUND}

The frontier of mesoscale science is rife with opportunities for discovery [1], with the ultimate goal of linking atomistic, unit processes with component-level performance. Since its inception [2], those interested in mesoscale science seek to understand the mechanistic reasons for the evolution of material properties not just from defects, but also from defect populations, superstructures, and spatiotemporal variations. However, direct, accurate theoretical predictions of mesoscale material properties remains difficult. Theory often treats the response functions for material properties as a superposition of the pertinent response functions for a homogeneous medium and a mathematically convenient distribution of single defects. Analytical treatment of a complex material, characterized by a variety of defect types, sizes, and spatial distributions, is beyond current theory. In these cases experimental measurements can provide insights into the mesoscale defect structures of such a material, but direct measurements remain difficult to perform or even conceive.

Ideally, one would use theory to inform the development of an experiment designed to elucidate the response mechanism

\footnotetext{
*hereiam@mit.edu

Published by the American Physical Society under the terms of the Creative Commons Attribution 4.0 International license. Further distribution of this work must maintain attribution to the author(s) and the published article's title, journal citation, and DOI.
}

of interest, and then use simulations to confirm experimental results. Another potential path for success is to use simulations as a predictive tool, validated by theory, to simultaneously guide and understand experimental measurements. For a field without a fully described theoretical framework, such as mesoscale radiation materials science, this second path is the only viable one. However, it cannot proceed without first rigorously validating the simulation framework using theoretical predictions and well-understood experimental measurements. In the absence of fully developed theory, predictive simulations and experimental measurements must be able to directly show radiation-induced changes in material properties over mesoscale (tens to hundreds of micron) volumes, with enough sensitivity to exclude confounding effects.

In radiation materials science, quantities such as the Young's modulus must be reliably determined to hundreds of parts per million (ppm), as radiation-induced defects change elastic properties in well-defined, though small, amounts. Vacancies have been analytically and computationally shown to change elastic moduli on the same order of magnitude as their concentration $[3,4]$. Interstitial atoms are predicted to have an effect between two [3] and ten [4-6] times as strong. Thermal equilibrium populations of defects are found in levels from $10^{-10}$ to $10^{-4}$ atom fraction for vacancies from room temperature to the melting point, respectively, and at levels thousands of times lower for interstitials. Radiation damage can increase vacancy fractions to a theoretical maximum of one percent during intense irradiation [7]. Direct, ultrasonic measurements of defect effects on elastic moduli have been carried out by several authors [8-10]. Irradiation can also 
create dislocations, which nonlinearly affect the Young's modulus as compared to a perfectly annealed material [11]. High-dose irradiation may also induce void swelling, which is expected to reduce the Young's modulus by a magnitude on the order of the void volume fraction [12]. In all cases, the ability to resolve changes of $1 \%$ and smaller in the elastic constants is necessary in a mesoscale measurement technique sensitive to these properties for radiation damage.

One promising tool for these direct, mesoscale measurements is transient grating (TG) spectroscopy [13,14]. This technique optically induces and monitors monochromatic surface acoustic waves (SAWs) with micron-scale wavelengths over an area on the order of $0.01 \mathrm{~mm}^{2}$. Characterization of these waves allows direct mesoscale measurement of elastic constants, thermal diffusivity, and acoustic damping. Robust repeatability of these measurements at the necessary sensitivity level is required to accurately analyze irradiation-induced changes to material properties. These material properties can be anisotropic in crystalline materials, such that knowing the precise surface-normal orientation and relative rotation of the crystal face (for single crystals) or grain size and texture (for polycrystals) is critical to the sensitivity of the measurement.

Elastic anisotropy causes the SAW speed to vary as a function of relative orientation on single-crystal surfaces. Here, anisotropy is defined for cubic crystals by the Zener anisotropy ratio, $A$, as

$$
A=\frac{2 C_{44}}{\left(C_{11}-C_{12}\right)},
$$

where $C_{i j}$ are the components of the reduced elastic stiffness tensor in Voigt notation [15]. By rotating the orientation of induced SAWs on single-crystal surfaces, one can measure a change in SAW speed. This variation provides a convenient method of validating both the sensitivity of the experimental technique and a simulation framework using molecular dynamics (MD). The ultimate sensitivity test is to select materials with very low anisotropy, such as aluminum $(A=1.22)$, resulting in expected changes in SAW speed of fractions of a percent per degree of rotation. Only tungsten $(A=1.01)$ has a lower anisotropy ratio [16]. The speed of measured SAWs, $c_{R}$, can be shown to scale as

$$
c_{R} \propto \sqrt{E}
$$

where $E$ is an effective elastic modulus for the direction and crystal orientation under investigation [17]. Orientation-based TG measurements made on single-crystal metallic samples can thus serve two purposes. First, they can be used to qualify the TG technique for the study of fine changes in effective elastic modulus. Second, they can be used to set a lower bound on the systematic sensitivity of the TG spectroscopy technique. In addition, correctly simulating these orientation-based changes in the acoustic response will validate classical MD on the tens of nanometers scale as a predictive tool for such small changes in effective elastic modulus.

In [18], TG spectroscopy was proposed as a methodology for in situ property monitoring during ion beam irradiation. In this work, we show that transient grating (TG) spectroscopy is sensitive to changes in SAW speed on the order of changes expected as a result of irradiation. We then hypothesize that classical MD, scaled to an appropriate simulation size, can predict the linear elastic response of materials by periodically exciting a simulation volume analogously to the TG experiment and monitoring the dynamic response. A lower size limit of $\sim 50 \mathrm{~nm}$ for $\mathrm{MD}$ simulations is shown to retain excellent scaling in SAW speed with experiments. This imparts confidence in the ability of classical MD to predict the response of materials with mesoscale defect populations, such as those due to irradiation, where theory cannot yet describe them. As a proof of concept, the reduction in SAW speed with increasing vacancy fractions, up to the theoretical maximum of $X_{v}=10^{-2}$, is simulated in single-crystal copper and aluminum.

\section{METHODS}

In the following sections, examples of the measurement, simulation, and analysis methods are given for explanation. All raw data files, processing scripts, output data sets, simulation input files, intermediate relaxed atom files, final atomic configurations, compiled/processed data files, and the code used to generate the slowness surfaces with input parameters are hosted permanently on our GitHub repository [19].

\section{A. Experiment}

Experimental TG measurements are carried out on aluminum $(\mathrm{Al})$ and copper $(\mathrm{Cu})$ single crystals with $\{001\}$ and $\{111\}$ surface orientations purchased from the MTI Corporation. Aluminum samples are $>99.99 \%$ pure, mechanically polished to $<10 \mathrm{~nm}$ surface roughness, and have surface orientations within $\pm 2^{\circ}$ of the given index. Copper samples are $>99.99 \%$ pure, mechanically polished to $<3 \mathrm{~nm}$ surface roughness, and have surface orientations within $\pm 2^{\circ}$ of the given index. An $\{001\}$ oriented sample of $99.99 \%$ pure tungsten $(\mathrm{W})$ with $\pm 0.1^{\circ}$ misorientation was used as a calibration standard for all measurements.

The photoacoustic excitation and detection process used in TG spectroscopy proceeds as follows. Two excitation (pump) laser pulses are crossed at an angle $\theta$ at the surface of the sample under interrogation. These crossed beams project a spatially periodic intensity pattern, causing local, periodic thermal expansion with a fixed wavelength, $\Lambda$. This thermal excitation launches monochromatic, counterpropagating acoustic waves with wavelength $\Lambda$. A schematic of this excitation process is shown in Fig. 1. These acoustic waves may take on several characters depending on the elastic constants, density, and layered structure (in the case of film-substrate systems) of the sample being measured [13]. For samples of thickness $d_{\text {sample }} \gg \Lambda$, the excited acoustic waves may take on two possible characters, either surface acoustic waves (SAWs) or pseudo-surface acoustic waves (PSAWs). SAW propagation is modeled as the superposition of the three evanescent partial waves whose amplitude decays to zero at infinite depth into the sample. In contrast, PSAWs are comprised of two evanescent partial waves with a small bulk wave component which radiates energy away into the bulk of the sample [21]. Both SAW and PSAW character excitations are observed in this work. TG measurements for these experiments are carried out using a passively Q-switched, $532 \mathrm{~nm}$, solid-state laser with a pulse length of $300 \mathrm{ps}$, repetition rate of $1 \mathrm{kHz}$, spot size of $90 \mu \mathrm{m}$, 
(a)

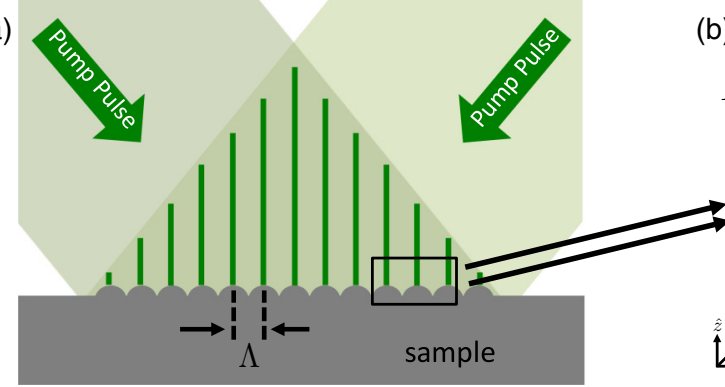

(b)

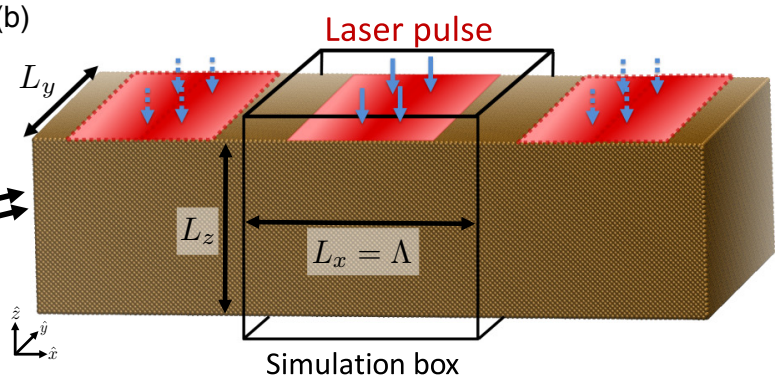

FIG. 1. Schematic of the induced surface excitation in experiment (a) and simulation (b). In experiment, crossed laser pulses create an interference pattern on the sample surface, causing spatially periodic heating and launching SAWs with fixed wavelength, $\Lambda$. A detailed description of the optical arrangement used in experiment can be found in [20]. In simulation, periodic boundary conditions are applied in all dimensions and a small vacuum gap is included within the periodic boundary in $\hat{z}$ to allow for the simulation of free surfaces. Emulation of the laser pulse is accomplished by increasing the temperature of atoms in the simulation periodically in the $\hat{x}$ direction.

and pulse energy at the sample surface of $2.15 \mu \mathrm{J}$ for $\mathrm{Al}$ samples and $1.75 \mu \mathrm{J}$ for $\mathrm{Cu}$ samples. Pumping power was reduced for $\mathrm{Cu}$ samples to avoid oxidizing the sample surface.

Detection of the induced acoustic waves is accomplished using an optical heterodyne detection technique [20]. In brief, a quasicontinuous wave probe laser is diffracted from the thermally induced displacement and refractive index variation on the sample surface [22]. This diffracted probe signal is heterodyne mixed with a spatially coincident reference beam and directed into a fast silicon avalanche photodetector with a $3 \mathrm{~dB}$ bandwidth of $1.0 \mathrm{GHz}$. The probe used in this experiment is a $785 \mathrm{~nm}, \mathrm{CW}$ diode laser modulated to a repetition rate of $1 \mathrm{kHz}$ with a $20 \%$ duty cycle, a power at the sample surface of $3.36 \mathrm{~mW}$, and a spot size of $150 \mu \mathrm{m}$. A more detailed description of an equivalent experimental setup can be found in [22]. The voltage output from the photodetector is recorded using a $5 \mathrm{GHz}$ bandwidth digital oscilloscope. Optical heterodyne detection increases the amplitude of the recorded traces by controlling the relative phase difference between probe and reference beams. For each measurement, two signals with a $180^{\circ}$ heterodyne phase offset are recorded and subtracted from each other in the analysis process. This allows for an increased signal-to-noise ratio and also allows for the removal of any long-time-scale detector response in the analyzed trace. A filtered power spectral density of these signals is taken and a peak-finding algorithm is used to determine the frequency of the dominant acoustic modes, $f_{T G}$, of each measurement [19]. A characteristic measured trace and its corresponding power spectrum can be seen in Fig. 2. The initial spike in signal amplitude corresponds to thermal impulse of the pulsed excitation. SAW oscillations are imposed over the background of the nonexponential decay of this thermal spike, which is fitted and subtracted before the power spectrum of the trace is calculated [23].

Angular-dependent measurements are taken using a manual rotation stage with $2^{\circ}$ gradations as a sample mount. All rotation measurements are carried out in air at ambient conditions using a nominal wavelength of $\Lambda=4.80 \mu \mathrm{m}$. As the actual projected transient grating spacing on the sample surface is a sensitive function of optical component placement [24], calibration measurements of the projected grating are taken before each measurement of interest. For calibration, a single crystal of $\{001\}$ oriented $\mathrm{W}$ is measured.
Since $\mathrm{W}$ is the least elastically anisotropic pure metal, with an expected variation in SAW velocity on the surface of $0.1 \%$, we use the experimentally determined SAW frequency, $f_{W}$, and a reference SAW speed on the $\{001\}$ surface of $W$ of $c_{W}=2665.9 \mathrm{~m} / \mathrm{s}$ to calculate the projected grating spacing for each measurement as

$$
\Lambda=\frac{c_{W}}{f_{W}} .
$$

The calculation of the speed $c_{W}$ was carried out using an isotropic approximation [17] with elastic constants aggregated from [25-27]. Using the value for the calibrated grating spacing, the acoustic wave velocity corresponding to peaks in the power spectrum can be calculated as $c_{T G}=\Lambda f_{T G}$.

Four single-crystal metallic samples are used in this study: $\{001\}$ and $\{111\}$ oriented $\mathrm{Al}$ and $\{001\}$ and $\{111\}$ oriented $\mathrm{Cu}$. For each sample under investigation data are collected by manually rotating the sample face in $5^{\circ}$ increments relative to the orientation of the induced SAW, as the absolute orientation of the SAW is fixed by the optical arrangement. The collection time for each measurement, averaging the response of 60000 laser pulses, is approximately five minutes. This time scale is primarily set by the necessity of manually changing the heterodyne phase once per measurement to be able to carry out background subtraction and signal amplification during processing.

\section{B. Simulation}

Molecular dynamics simulations using the LAMMPS (Large-scale Atomic/Molecular Massively Parallel Simulator) framework are used to emulate the SAW response that is observed in TG experiments [28]. Simulations are carried out on volumes with dimensions $L_{x} \times L_{y} \times L_{z}$, where the $\hat{x}$ axis is oriented along the direction of wave propagation and the $\hat{z}$ axis is along the incident direction of laser pulse. To mimic the TG experiment, we apply periodic boundary conditions in the directions of $\hat{x}$ and $\hat{y}$, whereas the top and bottom of the sample in $\hat{z}$ are free surfaces. The free surface is generated by making the atomic lattice thickness, $L_{z}$, slightly smaller than the periodic boundary in $\hat{z}$, creating a vacuum boundary of 5-6 nm between periodic cells in that dimension. In this case, the dimension $L_{x}$ represents the experimental transient grating wavelength $\Lambda$. The imposed MD boundary conditions 
(a)
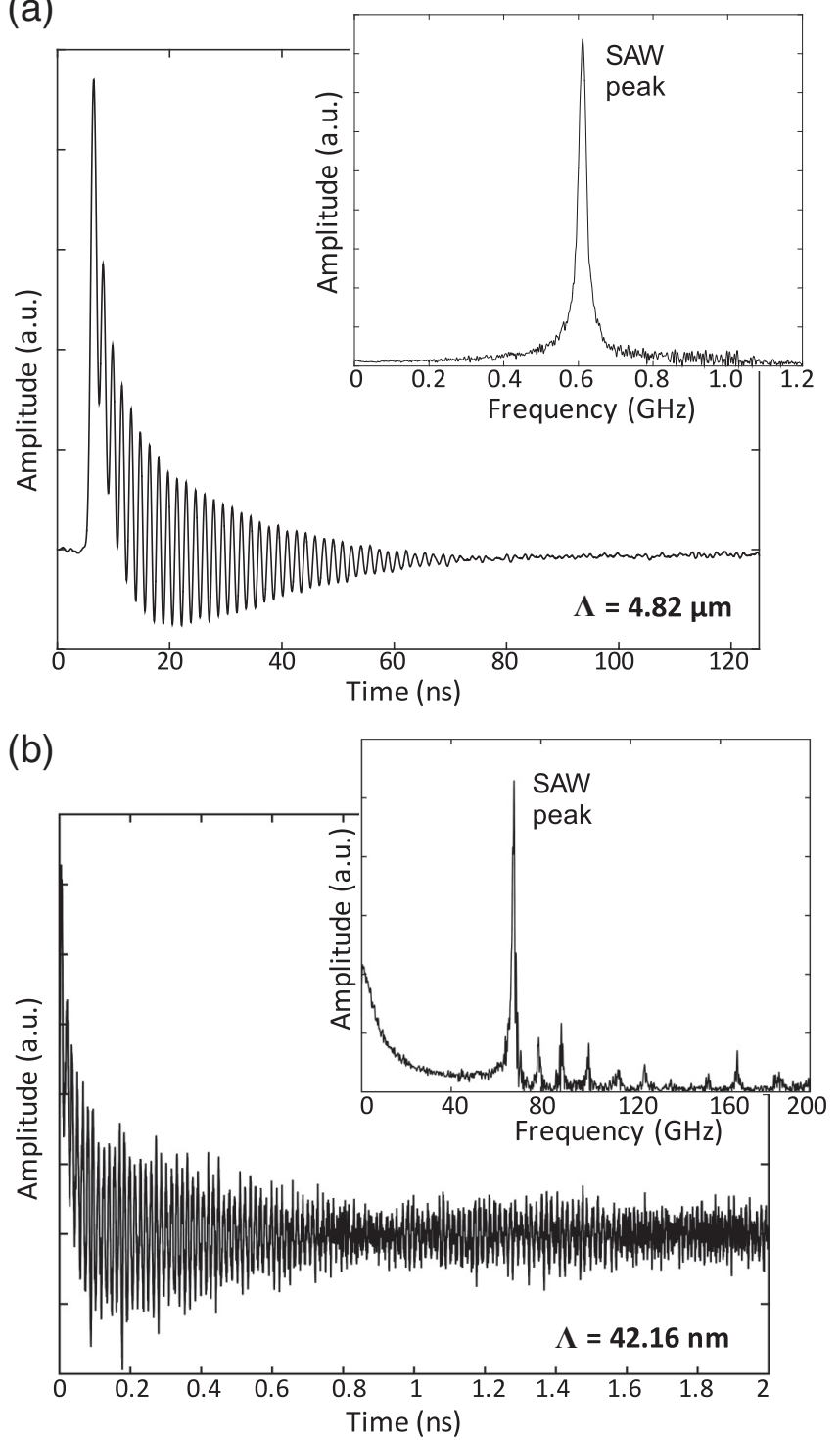

FIG. 2. Sample results from SAWs generated along the $\langle 100\rangle$ direction of $\{001\}$ oriented aluminum. Temporal traces and power spectra (inset plots) are shown for both experimental measurements (a) and MD simulations (b). The SAW wavelength used in experiment is $\Lambda=4.82 \mu \mathrm{m}$ and the simulated SAW propagation wavelength is $L_{x}=\Lambda=42.16 \mathrm{~nm}$.

and the relation of the simulation excitation scheme to the experimental case can be seen in Fig. 1. The wavelength effects on acoustic wave speed were studied by changing the sample length $L_{x}$ and will be discussed in detail in Sec. III C. Single crystals of $\mathrm{Al}$ and $\mathrm{Cu}$ with surface orientations $\{001\}$ and $\{111\}$ are investigated by rotating the simulation cell such that the appropriate crystal face appears normal to the surface. Embedded atom method (EAM) type interatomic potentials are employed for $\mathrm{Al}$ [29] and $\mathrm{Cu}$ [30], both of which are tuned using ultrasonically measured elastic constants. These EAM potentials have been shown to accurately describe elastic and thermal properties of these materials, though the elastic constants for the $\mathrm{Al}$ potential differ more from experimental values at room temperature than those for the $\mathrm{Cu}$ potential.
Before laser energy is imparted into the simulation, the system is relaxed at zero pressure for $20 \mathrm{ps}$ in the NPT ensemble (constant number of atoms, pressure, and temperature) and then for another $20 \mathrm{ps}$ in the NVT ensemble (constant number of atoms, volume, and temperature). The Nosé-Hoover thermostat is used in time integration and the time step is set to be 2 fs. This twice-relaxed lattice is used as the input for the laser-induced surface wave simulation, which is performed in the NVE (constant number of atoms, volume, and energy) ensemble.

The laser heating process is modeled using the Langevin thermostat by scaling the temperature of atoms as

$$
T(x, z, t)= \begin{cases}T_{0}+\delta T\left(\frac{t}{t_{0}}\right) \exp \left(\frac{-z}{z_{0}}\right), & |x| \leqslant x_{0}, \\ T_{0}, & |x|>x_{0},\end{cases}
$$

where $t_{0}$ is the laser pulse duration, $T_{0}$ is the initial simulation temperature (at $t=0$ ), taken as $300 \mathrm{~K}, \delta T$ is the maximum temperature increase at the end of the laser pulse (time $t_{0}$ ) and the sample surface, $z$ is the depth into the material, $z_{0}$ is the electron conduction depth (here taken as $7.5 \mathrm{~nm}$ [31-33]), $x$ is the distance along the wave propagation direction, and $x_{0}$ is the half-width of the excitation pulse. For all simulations with $L_{x}>15 \mathrm{~nm}$, the laser pulse, incident on the sample surface in the $\hat{z}$ direction, has a fixed width of $15 \mathrm{~nm}$ such that $x_{0}=7.5 \mathrm{~nm}$. For simulations with $L_{x}<15 \mathrm{~nm}$, the pulse has a width of $L_{x} / 2$ such that $x_{0}=L_{x} / 4$. Simulations with a Gaussian applied temperature profile in the $\hat{x}$ dimension were also tested with the $\mathrm{Cu}$ potential, but the resulting acoustic spectrum was unchanged from the uniform profile described above. After the heating process is complete, the simulation is allowed to relax in the $N V E$ ensemble.

The oscillations of the induced acoustic waves are recorded by tracking the $\hat{z}$ position of the center of mass of two small patches of atoms on the free surface of the simulation, one at the center of simulation volume and one located along the periodic boundary, $L_{x} / 2$ away in the $\hat{x}$ direction from the first patch. The acoustic oscillations of interest, those with periodicity $L_{x}$, are $180^{\circ}$ out of phase between these two patches of atoms. By subtracting the $\hat{z}$ center-of-mass position of the second patch from the first, a representative trace can be generated from simulations which is equivalent to the photodetector signal recorded in experiment. The power spectrum of this trace can similarly be used to isolate the excited acoustic modes using processing identical to that used for experimental data. A representative trace from a simulation is shown in Fig. 2(b) along with the associated power spectrum as a comparison to the trace collected in an experiment.

Unlike experiments, for simulations $d_{\text {sample }} \sim \Lambda$ and as a consequence it is possible that the primary acoustic oscillation may not be at the same velocity as the SAWs observed in experiment. In this thin-film regime the lowest-order symmetric and antisymmetric Lamb modes have not yet collapsed to the Rayleigh wave velocity at which we expect to observe SAW oscillations in "bulk" experimental samples; see Fig. 11 in [13]. Therefore, a series of simulations are conducted to determine an appropriate simulation thickness, $L_{z}$, such that the Lamb modes have collapsed and the simulated SAW speed no longer varies as a function of thickness. These simulations show that the SAW and PSAW speeds do not vary once $L_{z}>1.5 L_{x}$. 
Therefore, all the results presented in this work are obtained by setting sample thickness $L_{z}=2 L_{x}$, such that the simulation is thick enough to capture the SAW response while maintaining a small simulation volume to minimize computation time.

\section{Analytical SAW velocity calculation}

The expected velocity of acoustic waves excited in TG experiments can be calculated by considering the elastic wave equation

$$
\rho \frac{\partial^{2} u_{j}}{\partial t^{2}}=C_{i j k l} \frac{\partial^{2} u_{k}}{\partial x_{i} \partial x_{j}},
$$

where $u_{i}$ are the components of the displacement vector, $x_{i}$ are the Cartesian axes, and $C_{i j k l}$ is the elastic stiffness tensor of the material in question. Solutions to this equation are sought in the form of plane waves

$$
u_{i}=U_{i} \exp [i(\boldsymbol{k} \cdot \boldsymbol{x}-\omega t)]
$$

where $U_{i}$ are the components of the polarization vector, $\boldsymbol{k}$ is the wave vector (with direction parallel to the wave normal), and $\omega$ is the angular frequency [34]. A useful method for exploring surface wave phenomena obeying this set of wave propagation equations is the Fourier-domain surface dynamic Green's function $G_{i j}\left(\boldsymbol{k}_{\|}, \omega\right)$ [21]. This function describes the $i$ th component of the displacement response of a surface to a force acting in the $j$ direction as a function of the wave vector on the surface of the material, $\boldsymbol{k}_{\|}$, and the frequency $\omega$.

To a good approximation, measurements made using TG spectroscopy are sensitive to displacements normal to the surface of a semi-infinite half-space induced by a forcing function normal to the surface. As the excitation depth of acoustic waves in the TG experiment is approximated by the acoustic wavelength, $\Lambda$, this semi-infinite approximation is appropriate as long as the thickness of the sample is much greater than $\Lambda$. To calculate the acoustic wave speeds expected in an experiment, we consider an infinite half-space normal to $x_{3}$ occupying the domain $x_{3}>0$. By considering a force acting normal to this surface, the Green's function $G_{33}\left(\boldsymbol{k}_{\|}, \omega\right)$ can be calculated, as detailed in [21]. Allowed surface wave modes appear as poles in $G_{33}$ and can be visualized by plotting intensity maps of $\operatorname{Im}\left[G_{33}\right]$ as a function of the acoustic slowness (or inverse velocity), $\boldsymbol{s}_{\|}=\boldsymbol{k}_{\|} / \omega$. These "slowness surface" calculations can be carried out for materials of arbitrary crystal symmetry and surface orientation by proscribing the appropriate values for $C_{i j k l}$ and rotating the stiffness tensor such that the desired face is normal to $x_{3}$. All calculations of $\operatorname{Im}\left[G_{33}\right]$ presented in this work are conducted using an algorithm detailed in [21].

\section{RESULTS}

Results from both TG experiments and simulations on $\mathrm{Al}$ and $\mathrm{Cu}$ are presented in Fig. 3, overlaid on the slowness surfaces calculated with the reference elastic constant values in Table I for each of the four material and orientation combinations tested. The features of both sets of results will be discussed in turn.
TABLE I. Reference values of reduced elastic constants and Zener anisotropy ratio, $A$, used in analytic calculation of acoustic wave speeds as well as the values given from the EAM potentials used for MD simulations at $325 \mathrm{~K}$ for $\mathrm{Al}$ and $300 \mathrm{~K}$ for $\mathrm{Cu}$.

\begin{tabular}{lcccc}
\hline \hline & $C_{11}(\mathrm{GPa})$ & $C_{12}(\mathrm{GPa})$ & $C_{44} \mathrm{GPa}$ & $A$ \\
\hline $\mathrm{Al}$ (ref.) [16] & 107.3 & 60.9 & 28.3 & 1.22 \\
$\mathrm{Cu}$ (ref.) [35] & 168.4 & 121.4 & 75.4 & 3.23 \\
$\mathrm{Al}$ (MD) [29] & 107.7 & 57.9 & 26.2 & 1.05 \\
$\mathrm{Cu}(\mathrm{MD})[30]$ & 169.9 & 122.6 & 76.2 & 3.23 \\
\hline \hline
\end{tabular}

\section{A. Theoretical predictions}

Calculated slowness surfaces, including overlaid experimental and simulation results, for the $\{001\}$ and $\{111\}$ crystal surfaces of both $\mathrm{Al}$ and $\mathrm{Cu}$ are shown in Fig. 3. Reference values for the reduced elastic constants used for the calculation presented in Fig. 3 as well as the constants given from the MD potentials are listed in Table I.

In intensity plots of $\operatorname{Im}\left[G_{33}\right]$ the poles corresponding to the Rayleigh-character SAWs appear as thin, dark lines bounding the bulk of the slowness surface. For very anisotropic materials such as $\mathrm{Cu}$ there are certain directions on certain crystal planes at which the polarization of the SAW can change from out-of-plane (along $x_{3}$ ) to in-plane (along $x_{1}$ and $x_{2}$ ). In these cases, the response is not captured in $G_{33}\left(\boldsymbol{k}_{\|}, \omega\right)$. This can be seen in the right of Fig. 3 on the $\{001\}$ surface near the $\langle 110\rangle$ direction as the intensity of the SAW fades. PSAWs appear on these surfaces as similar dark bands, but at smaller values of slowness, and therefore higher speed, than the SAW excitations. The remainder (lighter gray areas) of these surfaces represent bulk acoustic modes captured in $G_{33}\left(\boldsymbol{k}_{\|}, \omega\right)$, but not excited in the TG experiments.

\section{B. Experimental results}

The range of experimental data collection for each sample is chosen to match the periodicity of the expected acoustic response, namely over a range of $90^{\circ}$ for $\{001\}$ oriented samples and $60^{\circ}$ for $\{111\}$ oriented samples. Measured SAW speeds are inverted and projected along their relative directions to be plotted on the slowness surface such that each data point has coordinates given by

$$
\left(s_{1}, s_{2}\right)=\frac{1}{c_{T G}}(\sin \theta, \cos \theta),
$$

where $\theta$ is the relative orientation of the induced acoustic wave vector on the sample surface. As the surface directions of the crystals is not known a priori, the data presented in Fig. 3 include an arbitrary phase shift with respect to the $\langle 100\rangle$ or $\langle 1 \overline{1} 0\rangle$ directions such that the maxima and minima of each data set are aligned with the correct position on the slowness surface.

The relative error in each SAW speed measurement, inclusive of that incurred by determining the grating spacing, $\Lambda$, with a tungsten calibration sample and of the finite width of the peaks in the TG power spectra, is on the order of $0.1 \%$ based on measured values. This order holds true for all experimental measurements taken as part of this study on both $\mathrm{Al}$ and $\mathrm{Cu}$. 
(a)

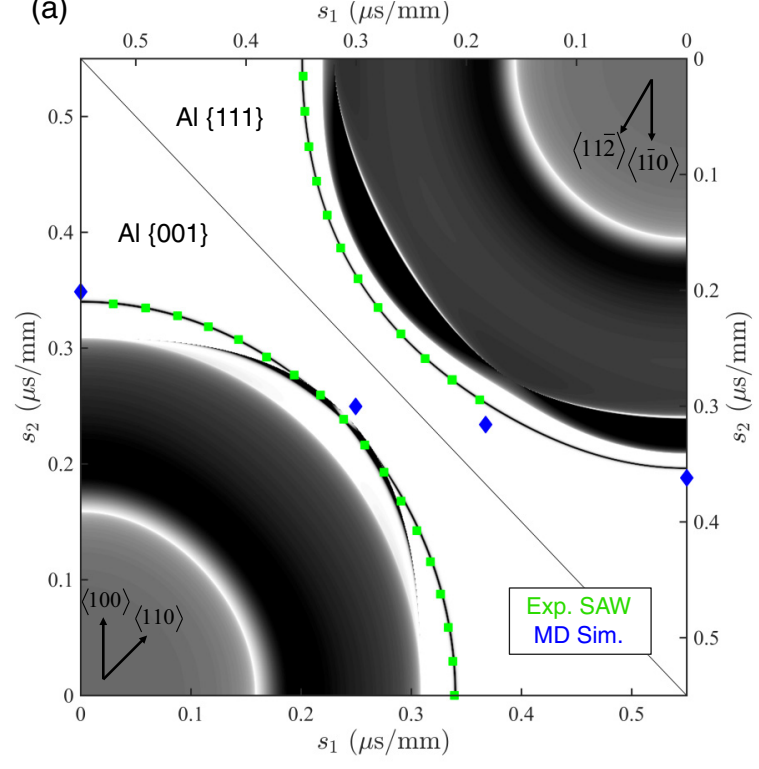

(b)

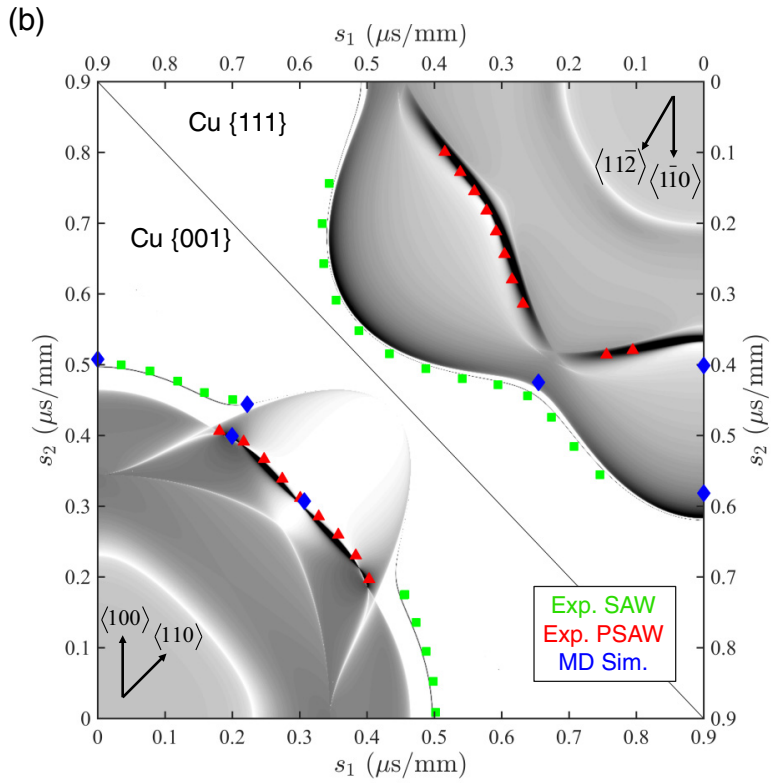

FIG. 3. Comparison of theoretical, experimental, and simulated acoustic slownesses for $\{001\}$ and $\{111\}$ oriented single crystals of (a) aluminum and (b) copper. Primary directions on each crystal surface are indicated by arrows in the corners of each map. The gray-scale contour maps show $\operatorname{Im}\left[G_{33}\right]$ for each surface. The thin, dark lines bounding each surface correspond to the surface acoustic mode and dark lines farther toward the origin indicate pseudo-surface acoustic modes. Experimental measurements corresponding to SAWs are labeled with green squares and experimental measurements corresponding to PSAWs are labeled with red triangles. Simulated SAW and PSAW slownesses are indicated with blue diamonds along primary directions. Experimental measurements match predicted wave slownesses in both magnitude and periodicity for all cases considered. Simulations capture both the SAW and PSAW behavior well for $\mathrm{Cu}$, but less well for Al.

The error incurred due to finite width peaks is taken as the 95\% confidence interval on the value of the peak location parameter for each peak in a TG power spectrum. The width of these peaks is consistently on the order of $15 \mathrm{MHz}$. The error bars on each measurement are not included in Fig. 3 as they are virtually indistinguishable given the marker size on the plot. The order of magnitude of the relative error makes the 1\%-2\% change in SAW speed along different directions on the $\mathrm{Al}$ crystals easily distinguishable in the experimental measurements.

The small deviation in the absolute measured values from the values predicted using $\operatorname{Im}\left[G_{33}\right]$, for example along $\{111\}\langle 11 \overline{2}\rangle$ in $\mathrm{Cu}$, is expected given the sensitivity of the calculation to the particular reference values chosen for the elastic constants. However, the expected periodicity is matched in experiment in all cases presented. Even for the case where the absolute agreement appears the worst, on the $\{111\}$ surface of $\mathrm{Cu}$, the ratio of the maximum measured SAW speed (along $\langle 11 \overline{2}\rangle$ ) to the minimum measured SAW speed (along $\langle 1 \overline{1} 0\rangle$ ) is 1.2171 where the values from the reference calculation give a ratio of 1.2178, giving a worst-case experimental error of $575 \mathrm{ppm}$. Given the level of agreement between calculated and measured slowness values, these experiments are clearly an accurate reflection of the physical properties of the single crystals under investigation.

\section{Simulation results: Pure materials}

In addition to the convergence study used to determine the minimum appropriate value of $L_{z}$, a set of simulations is car- ried out varying $L_{x}$ to determine the minimum acoustic wavelength necessary to accurately capture the experimental SAW response. These simulations are oriented along the $\{001\}\langle 100\rangle$ and $\{001\}\langle 110\rangle$ directions using the aforementioned EAM Al potential. Figure 4 shows simulated and measured SAW speeds for the $\{001\}\langle 100\rangle$ direction over several orders of magnitude in excitation wavelength. Simulations are run with wavelengths ranging from $2.4 \mathrm{~nm}$ to $203 \mathrm{~nm}$; experimental measurements using $\Lambda$ from 2.05 to $4.82 \mu \mathrm{m}$ are included as a reference and illustrate the excellent scaling behavior of the simulations. As is clear from Fig. 4, at very small $L_{x}$ the continuum elasticity approximation that is used to calculate SAW speeds breaks down. This approximation is valid for the mesoscale excitations that are being induced in experiment, but surface phonon dispersion effects become apparent in small simulations [36]. In the range of $L_{x}=40-100 \mathrm{~nm}$, simulations have a small and fairly consistent deviation from the calculated SAW speed in that direction. Therefore, for all other simulations besides those on $\{001\} \mathrm{Al}$, a target wavelength of $40-50 \mathrm{~nm}$ is chosen for expedited computation time without sacrificing accuracy.

For both $\mathrm{Al}$ and $\mathrm{Cu}$, the following crystal orientation and directions are simulated: $\{001\}\langle 100\rangle,\{001\}\langle 110\rangle,\{111\}\langle 1 \overline{1} 0\rangle$, and $\{111\}\langle 11 \overline{2}\rangle$. These directions represent the expected maximum and minimum SAW, and PSAW in the case of $\mathrm{Cu}$, speeds for these cubic crystal orientations. In addition, the $\{001\}\langle 120\rangle$ direction is simulated for $\mathrm{Cu}$ for further validation of the methodology. As these simulations are processed in the same manner as experimental TG measurements, the resultant SAW speeds are also converted to vector slownesses using 


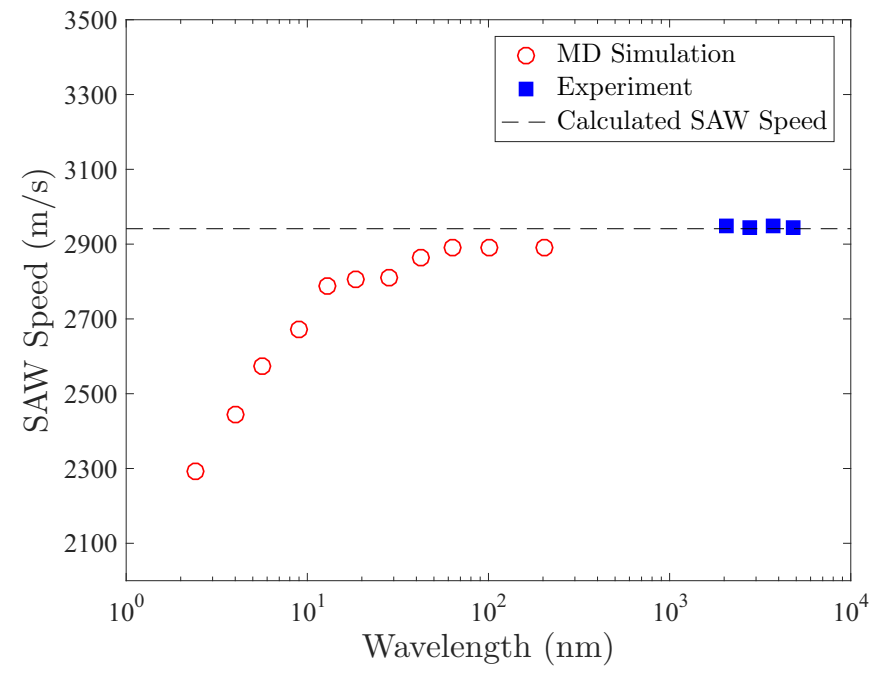

FIG. 4. Dependence of the measured SAW velocity in experiment (blue squares) and simulation (open red circles) on wavelength for SAWs induced along the $\langle 100\rangle$ direction on $\{001\}$ crystals of aluminum. The analytically predicted SAW speed for this orientation and direction, $2941.2 \mathrm{~m} / \mathrm{s}$, is plotted as the dashed line. At very small wavelengths there is a significant deviation between the predicted and simulated SAW speeds.

Eq. (7) and are plotted on Fig. 3. All of the simulation data points on Fig. 3 use wavelengths in the range specified above.

As is apparent from Fig. 3, the simulated TG response agrees very well with the calculated and measured SAW and PSAW speeds for the $\{001\}$ orientations of both $\mathrm{Al}$ and $\mathrm{Cu}$. The additional $\langle 120\rangle$ direction on the $\mathrm{Cu}$ surface shows that not only are the magnitudes of the responses close to the expected values, but also the simulations are able to capture the dynamic response on intermediate crystal directions. It is noteworthy that this intermediate behavior is captured accurately even along a direction at which the polarization of the SAW has almost undergone the transition from normal-to-the-surface to parallel-to-the-surface and both SAW and PSAW modes are identifiable in the response.

As Fig. 3 also shows, the simulated responses match less well on the $\{111\}$ surfaces on both materials. The mismatch, which overpredicts SAW speeds on $\mathrm{Cu}$ and underpredicts SAW speeds on $\mathrm{Al}$ and the PSAW speed on $\mathrm{Cu}$, is likely a result of the potentials used in the MD simulation failing to capture an accurate elastic response on a noncubic crystal plane. To explore this mismatch further, the set of reduced elastic constants given by the validated MD potentials, as listed in Table I, is used as an input into the calculation of $\operatorname{Im}\left[G_{33}\right]$. If the set of constants given by these potentials produces SAW and PSAW speeds significantly different from those given by our reference set of constants, it is possible that the TG simulations are accurately representing the dynamics governed by the MD potentials while disagreeing with experiment and reference calculations. Although the constants given by the $\mathrm{Cu}$ potential closely match those of the reference set, the $C_{i j}$ values for the Al potential differ noticeably. Most importantly for the surface direction variation in the acoustic response, the Zener anisotropy ratio for the $\mathrm{Al}$ potential $(A=1.05)$ is significantly different from the reference case $(A=1.22)$.

Table II shows the calculated SAW and PSAW speeds for the primary directions of both orientations and types of crystal investigated using both the reference constants, labeled "ref.", the constants given by the potentials from [29] and [30], labeled "MD calc.", as well as the percent difference of the MD calc. value from the reference value. Additionally, the results found by running the TG simulation process are listed as "MD sim.". As expected given the values in Table I, the acoustic speeds calculated from the Al potential differ more significantly from the reference case than those for $\mathrm{Cu}$. The values for acoustic wave speeds from calculations using the MD potential elastic constants are, in all cases, closer to the calculated values using the reference constants than the results of the TG simulations.

\section{Simulation results: Materials with defects}

Finally, a series of simulations along the $\{001\}\langle 100\rangle$ direction in both $\mathrm{Al}$ and $\mathrm{Cu}$ is carried out with an imposed vacancy fraction $X_{v}$ ranging from $10^{-4}$ to $10^{-2}$ with initial size $L_{x}=42.7 \mathrm{~nm}$ for $\mathrm{Al}$ and $47.5 \mathrm{~nm}$ for $\mathrm{Cu}$. Simulations of this nature are the first real attempt at validating this type of approach, as is it not possible to make reference calculations of the acoustic response for materials whose elastic constants are not known a priori. The construction process for these simulations proceeds as follows:

TABLE II. Calculations and simulations of acoustic wave speed for $\{001\}$ and $\{111\}$ orientations on $\mathrm{Al}$ and $\mathrm{Cu}$. Speeds from calculations using the reference values for elastic constants (ref.), using the values of elastic constants from MD potentials (MD calc.), and from MD transient grating simulations (MD sim.) are all given in $\mathrm{m} / \mathrm{s}$. The \% Diff. column for each material is the deviation of the MD calc. value from the reference value.

\begin{tabular}{|c|c|c|c|c|c|c|c|c|}
\hline Direction & $\begin{array}{c}\text { Al } \\
\text { (ref.) }\end{array}$ & $\begin{array}{c}\mathrm{Al} \\
(\mathrm{MD} \text { calc. })\end{array}$ & $\begin{array}{c}\mathrm{Al} \\
(\mathrm{MD} \text { sim.) }\end{array}$ & $\begin{array}{c}\mathrm{Al} \\
(\% \text { Diff.) }\end{array}$ & $\begin{array}{c}\mathrm{Cu} \\
\text { (ref.) }\end{array}$ & $\begin{array}{c}\mathrm{Cu} \\
\text { (MD calc.) }\end{array}$ & $\begin{array}{c}\mathrm{Cu} \\
(\mathrm{MD} \operatorname{sim} .)\end{array}$ & $\begin{array}{c}\mathrm{Cu} \\
(\% \text { Diff.) }\end{array}$ \\
\hline$\{001\}\langle 100\rangle$ SAW & 2941.2 & 2893.5 & 2868 & -1.62 & 2011.7 & 2018.6 & 1970 & +0.34 \\
\hline$\{001\}\langle 110\rangle$ SAW & 2968.5 & 2898.0 & 2833 & -2.37 & - & - & - & - \\
\hline$\{001\}\langle 110\rangle$ PSAW & - & - & - & - & 2340.6 & 2350.8 & 2303 & +0.44 \\
\hline$\{111\}\langle 110\rangle$ SAW & 2826.5 & 2867.8 & 2762 & +1.46 & 1613.9 & 1618.4 & 1719 & +0.28 \\
\hline$\{111\}\langle 1 \overline{1} 0\rangle$ PSAW & - & - & - & - & 2755.6 & 2774.7 & 2493 & +0.69 \\
\hline$\{111\}\langle 11 \overline{2}\rangle$ SAW & 2865.3 & 2871.9 & 2740 & +0.23 & 1965.4 & 1970.1 & 2037 & +0.24 \\
\hline
\end{tabular}




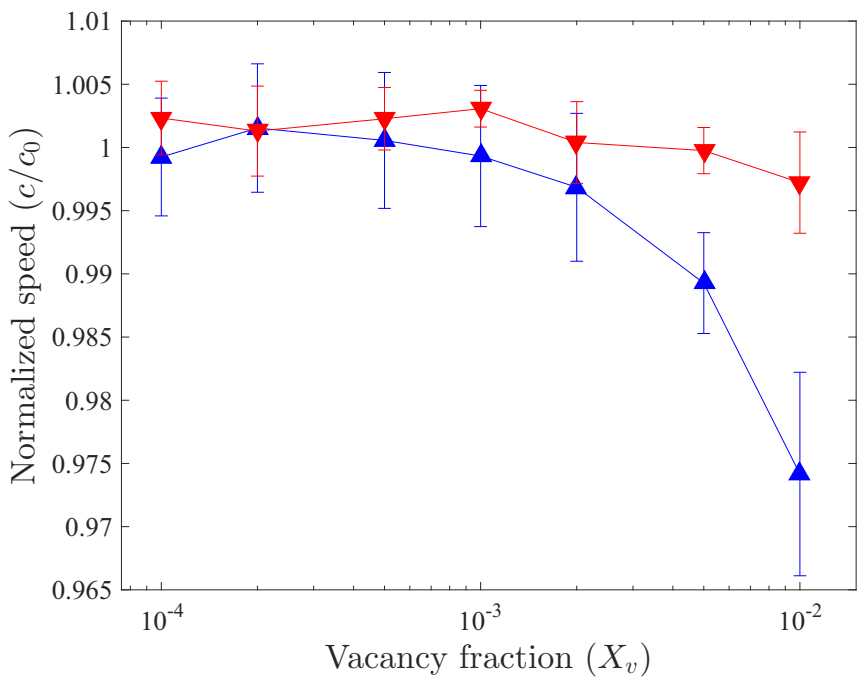

FIG. 5. SAW speed along the $\{001\}\langle 100\rangle$ direction in $\mathrm{Al}$ (red) and $\mathrm{Cu}$ (blue), $c$, normalized to the perfect lattice speed, $c_{0}$, as a function of vacancy fraction, $X_{v}$, for each material.

(1) An initial volume of atoms is created with the specified wavelength and orientation.

(2) A number of atoms necessary to give the desired value of $X_{v}$ are randomly deleted from the lattice.

(3) The simulation is allowed to relax at zero pressure in the $N P T$ ensemble.

(4) The relaxed value of $L_{x}$, smaller than the initialized value, is recorded.

(5) The heating and relaxation process proceeds as normal.

Recording the value $L_{x}$ after $X_{v}$ is imposed and the lattice relaxed is necessary to accurately calculate the resultant SAW speed from the simulation's power spectrum. The $\{001\}\langle 100\rangle$ direction is chosen for these simulations because simulations in this direction closely matched both reference calculations and experiments for $\mathrm{Cu}$ and $\mathrm{Al}$ in perfect crystals. Figure 5 shows the relative change in SAW speed compared to the perfect crystal baseline, as reported in Table II, for both of these cases. For each point on this plot, eight to ten simulations were carried out with different random configurations of defects, and the speed for each was calculated using the wavelength of the defect-relaxed lattice. The error bars represent one standard deviation between the simulation sets for each $X_{v}$. An overall reduction in SAW speed as $X_{v}$ increases is clear for the $\mathrm{Cu}$ case, although no statistically significant trend is evident for Al.

\section{DISCUSSION AND IMPLICATIONS}

There are two primary implications of this work. The major takeaway from the experimental results is that subpercent relative changes in acoustic wave speed are readily and repeatably detectable using the TG spectroscopy experiment in its current implementation. Given the scaling in Eq. (2), detecting subpercent changes in the effective elastic modulus is possible. The test problem considered here, the effect of single-crystal anisotropy on SAW speed in different directions, is a well-validated physical phenomenon. These benchmark measurements show that TG spectroscopy can be confidently used as methodology for detecting small changes in elastic properties as a response to different stimuli. In particular, the level of sensitivity shown here $(0.1 \%$ absolute, $<575 \mathrm{ppm}$ relative) is well within the bounds necessary to detect irradiationinduced material responses, such as volumetric void swelling. The detection of drastically increased point defect populations in irradiated materials should also be experimentally possible, and future work will be focused on disentangling the effects of different types of defects on the TG response.

However, TG experiments on materials with elevated defect populations cannot be intelligently designed without some tool to predict the variation in response with defect concentration and character. Since the elastic properties of materials with arbitrary, spatially varying, defect populations are not known, the analytical framework for calculating the acoustic response of pure materials is not an appropriate predictive tool for this use.

Therefore, the second major implication of this work is that classical MD can be used to simulate an excitation and detection process very closely resembling experiments. Simulations have been shown to most appropriately capture the dynamics of mesoscale experiment using excitation wavelengths of $L_{x} \sim 50 \mathrm{~nm}$ with a thickness normal to the surface of $L_{z}=2 L_{x}$. Simulations with arbitrary crystal orientation and direction can be created and inserted into a general TG excitation framework, whose parameters have been optimized to provide the most accurate dynamic acoustic response possible with standard EAM potentials.

Throughout this work, the standard MD potentials for $\mathrm{Al}$ and $\mathrm{Cu}$ have been taken as a given. It is clear that the acoustic response is captured less well on $\{111\}$ oriented crystals than on $\{001\}$ oriented crystals and less well for $\mathrm{Al}$ than $\mathrm{Cu}$. This, however, is not a failing of the methodology, but rather a weakness of the potentials themselves. We are using these potentials, which have been validated on small-scale parameters such as atomic volume and phonon dispersion, to capture a much larger dynamic process. It is not unexpected, then, that there is some variation between calculated and experimentally measured acoustic responses and those we are able to simulate. It is remarkable, in fact, that mesoscale and even continuum dynamic processes are so well captured by these potentials. One possibility moving forward, given the flexibility of the TG simulation framework, is to use simulations of these dynamic responses as an additional validation tool for new interatomic potentials, particularly because the TG process captures more of the nonlinear predictions of potentials than simple checks of lattice parameter or elastic constants.

Table II shows that using elastic constants calculated using the classical MD potentials as inputs in the calculation of $\operatorname{Im}\left[G_{33}\right]$ provides values for SAW and PSAW speeds closer to those calculated from reference constants. This same procedure could be used with uniform defect populations, such as those used in this study, to predict the change in SAW speed with defect concentration. However, the larger-scale excitation technique that is detailed in this work has the advantage of being able to accommodate spatially varying defect populations. The ability to simulate the TG response of materials with a spatial distribution of defects similar 
to that induced from radiation damage, both from microheterogeneity as well as macro-heterogeneity specific to ion irradiation, will be necessary to use this methodology as a predictive tool moving forward.

The effects of increasing vacancy concentration in simulations gives insight into the magnitude of the change in SAW speed that should be expected under irradiation. For these simulations a crystal orientation and direction whose response agrees well with the predicted response for perfect materials is used. In $\mathrm{Cu}$, the reduction in speed is about $3 \%$ for a $1 \%$ vacancy concentration. However, for $\mathrm{Al}$, the reduction is only about $0.5 \%$. In the limit of small changes in elastic moduli, the scaling in Eq. (2) indicates that the change in SAW speed should scale as $\Delta c_{R} / c_{R} \approx \Delta E / 2 E$. Using this simple scaling with previous ultrasonically measured stiffness changes, the expected change in $c_{R}$ for $X_{v}=0.01$ for $\mathrm{Cu}$ is $3.5 \%$ and for $\mathrm{Al}$ is $0.75 \%[9,10]$. Both previous experiments and our MD simulations show reductions consistent with theory and are within sensitivity limits of experimental TG detection [4]. In an effort to simulate $\mathrm{TG}$ responses representative of heterogeneous irradiation-induced defect populations, future work includes using MD to simulate damage cascades in materials, evolving these damaged structures using either cluster dynamics or kinetic Monte Carlo methods, and finally simulating the TG response with the MD methodology described in this work [37-39].

\section{CONCLUSIONS}

Given the overall sensitivity of transient grating experimental measurements and the ability to simulate the TG excitation and response using classical MD, this technique is well poised to be utilized as a mesoscale technique for identifying radiation-induced changes in material properties. However, much work remains to be able to confidently deconvolve the integral effects of the variety of defects expected as a result of radiation damage. Appropriate initial implementations of this technique would be for cases where a single microstructural effect dominates the change in thermal and mechanical properties, as in the case of volumetric void swelling. The simulation approach detailed here allows the effect of arbitrary defect populations on the dynamic acoustic response to be quantified. This will be an invaluable predictive tool for guiding future TG experiments on materials with isolated types of defect populations as well as irradiated materials. The work presented here moves us towards the development of TG spectroscopy as a noncontact, nondestructive methodology for in situ property monitoring as irradiation is taking place.

\section{ACKNOWLEDGMENTS}

This paper is based upon work supported by the NSF Graduate Research Fellowship under Grant No. 1122374 and NSF Grant No. CHE-1111557. The authors also thank Transatomic Power, Inc., for their support under Award No. 023875-001. M.P.S. acknowledges funding from the US Nuclear Regulatory Commission's MIT Nuclear Education Faculty Development Program under Grant No. NRC-HQ84-15-G-0045. This research made use of the resources of the High Performance Computing Cluster at Idaho National Laboratory, which is supported by the Office of Nuclear Energy of the US Department of Energy and the Nuclear Science User Facilities under Contract No. DE-AC07-05ID14517.
[1] J. L. Sarrao and G. W. Crabtree, Opportunities and advances in mesoscale science, Curr. Opin. Solid State Mater. Sci. 19, 201 (2015).

[2] R. B. Laughlin, D. Pines, J. Schmalian, B.P. Stojkovic, and P. Wolynes, The middle way, Proc. Natl. Acad. Sci. USA 97, 32 (2000).

[3] S. Chiesa, P. M. Derlet, S. L. Dudarev, and H. Van Swygenhoven, Atomistic calculation of elastic constants of alpha-iron containing point defects by means of magnetic interatomic potentials, J. Nucl. Mater. 386-388, 49 (2009).

[4] G. J. Dienes, A theoretical estimate of the effect of radiation on the elastic constants of simple metals, Phys. Rev. 86, 228 (1952).

[5] F. R. N. Nabarro, Effect of radiation on elastic constants, Phys. Rev. 87, 665 (1952).

[6] G. J. Dienes, Effect of radiation on elastic constants, Phys. Rev. 87, 666 (1952).

[7] R. S. Averback and K. L. Merkle, Radiation-annealing effects in energetic displacement cascades, Phys. Rev. B 16, 3860 (1977).

[8] L. E. Rehn, J. Holder, A. V. Granato, R. R. Coltman, and F. W. Young, Effects of thermal-neutron irradiation on the elastic constants of copper, Phys. Rev. B 10, 349 (1974).
[9] R. G. Folweiler and F. R. Brotzen, The effect of quenched-in vacancies on the elastic modulus of aluminum, Acta Metall. 7, 716 (1959).

[10] H. Dieckamp and A. Sosin, Effect of electron irradiation on Young's modulus, J. Appl. Phys. 27, 1416 (1956).

[11] J. Friedel, Anomaly in the rigidity modulus of copper alloys for small concentrations, Philos. Mag. 44, 444 (1953).

[12] I. I. Balachov, E. N. Shcherbakov, A. V. Kozlov, I. A. Portnykh, and F. A. Garner, Influence of radiation-induced voids and bubbles on physical properties of austenitic structural alloys, J. Nucl. Mater. 329-333 (Part A), 617 (2004).

[13] J. A. Rogers, A. A. Maznev, M. J. Banet, and K. A. Nelson, Optical generation and characterization of acoustic waves in thin films: Fundamentals and applications, Annu. Rev. Mater. Sci. 30, 117 (2000).

[14] F. Hofmann, D. Nguyen-Manh, M. R. Gilbert, C. E. Beck, J. K. Eliason, A. A. Maznev, W. Liu, D. E. J. Armstrong, K. A. Nelson, and S. L. Dudarev, Lattice swelling and modulus change in a helium-implanted tungsten alloy: X-ray micro-diffraction, surface acoustic wave measurements, and multiscale modelling, Acta Mater. 89, 352 (2015).

[15] C. Zener, Contributions to the theory of beta-phase alloys, Phys. Rev. 71, 846 (1947). 
[16] G. Simmons and H. Wang, Single-Crystal Elastic Constants and Calculated Aggregate Properties: A Handbook, 2nd ed. (MIT Press, Cambridge, MA, 1971).

[17] P. G. Malischewsky, Comparison of approximated solutions for the phase velocity of Rayleigh waves (comment on "characterization of surface damage via surface acoustic waves"), Nanotechnology 16, 995 (2005).

[18] M. P. Short, C. A. Dennett, S. E. Ferry, Y. Yang, V. K. Mishra, J. K. Eliason, A. Vega-Flick, A. A. Maznev, and K. A. Nelson, Applications of transient grating spectroscopy to radiation materials science, JOM 67, 1840 (2015).

[19] C. Dennett et al., http://dx.doi.org/10.5281/zenodo.61206.

[20] A. A. Maznev, K. A. Nelson, and J. A. Rogers, Optical heterodyne detection of laser-induced gratings, Opt. Lett. 23, 1319 (1998).

[21] A. G. Every, A. A. Maznev, W. Grill, M. Pluta, J. D. Comins, O. B. Wright, O. Matsuda, W. Sachse, and J. P. Wolfe, Bulk and surface acoustic wave phenomena in crystals: Observation and interpretation, Wave Motion 50, 1197 (2013).

[22] J. A. Johnson, A. A. Maznev, M. T. Bulsara, E. A. Fitzgerald, T. C. Harman, S. Calawa, C. J. Vineis, G. Turner, and K. A. Nelson, Phase-controlled, heterodyne laser-induced transient grating measurements of thermal transport properties in opaque material, J. Appl. Phys. 111, 023503 (2012).

[23] O. W. Käding, H. Skurk, A. A. Maznev, and E. Matthias, Transient thermal gratings at surfaces for thermal characterization of bulk materials and thin films, Appl. Phys. A 61, 253 (1995).

[24] A. Vega-Flick, J. K. Eliason, A. A. Maznev, A. Khanolkar, M. Abi Ghanem, N. Boechler, J. J. Alvarado-Gil, and K. A. Nelson, Laser-induced transient grating setup with continuously tunable period, Rev. Sci. Instrum. 86, 123101 (2015).

[25] F. H. Featherston and J. R. Neighbours, Elastic constants of tantalum, tungsten, and molybdenum, Phys. Rev. 130, 1324 (1963).

[26] R. Lowrie and A. M. Gonas, Single-crystal elastic properties of tungsten from $24^{\circ}$ to $1800^{\circ}$ C, J. Appl. Phys. 38, 4505 (1967).

[27] D. I. Bolef and J. De Klerk, Elastic constants of single-crystal Mo and $\mathrm{W}$ between $77^{\circ}$ and $500^{\circ}$ K, J. Appl. Phys. 33, 2311 (1962).

[28] S. Plimpton, Fast parallel algorithms for short-range molecular dynamics, J. Comput. Phys. 117, 1 (1995).
[29] J. M. Winey, A. Kubota, and Y. M. Gupta, A thermodynamic approach to determine accurate potentials for molecular dynamics simulations: Thermoelastic response of aluminum, Modell. Simul. Mater. Sci. Eng. 17, 055004 (2009).

[30] Y. Mishin, M. J. Mehl, D. A. Papaconstantopoulos, A. F. Voter, and J. D. Kress, Structural stability and lattice defects in copper: Ab initio, tight-binding, and embedded-atom calculations, Phys. Rev. B 63, 224106 (2001).

[31] J. I. Etcheverry and M. Mesaros, Molecular dynamics simulation of the production of acoustic waves by pulsed laser irradiation, Phys. Rev. B 60, 9430 (1999).

[32] B. J. Demaske, V. V. Zhakhovsky, N. A. Inogamov, and I. I. Oleynik, Ablation and spallation of gold films irradiated by ultrashort laser pulses, Phys. Rev. B 82, 064113 (2010).

[33] X. Wang and $\mathrm{X}$. Xu, Molecular dynamics simulation of heat transfer and phase change during laser material interaction, J. Heat Transfer 124, 265 (2002).

[34] G. W. Farnell, Properties of elastic surface waves, in Physical Acoustics, Vol. VI, edited by W. P. Mason and R. H. Thurston (Academic Press, New York, 1970), Chap. 3, pp. 109-166.

[35] W. C. Overton and J. Gaffney, Temperature variation of the elastic constants of cubic elements. I. Copper, Phys. Rev. 98, 969 (1955).

[36] G. Benedek, M. Bernasconi, V. Chis, E. Chulkov, P.M. Echenique, B. Hellsing, and J. Peter Toennies, Theory of surface phonons at metal surfaces: Recent advances, J. Phys.: Condens. Matter 22, 084020 (2010).

[37] D. J. Bacon and T. D. de la Rubia, Molecular dynamics computer simulations of displacement cascades in metals, J. Nucl. Mater. 216, 275 (1994).

[38] D. Xu, B. D. Wirth, M. Li, and M. A. Kirk, Defect microstructural evolution in ion irradiated metallic nanofoils: Kinetic Monte Carlo simulation versus cluster dynamics modeling and in situ transmission electron microscopy experiments, Appl. Phys. Lett. 101, 101905 (2012).

[39] D. Xu, B. D. Wirth, M. Li, and M. A. Kirk, Combining in situ transmission electron microscopy irradiation experiments with cluster dynamics modeling to study nanoscale defect agglomeration in structural metals, Acta Mater. 60, 4286 (2012). 